\title{
Erratum
}

\section{Theory of Optical Ramsey Resonance in Three Separated Fields Produced by a Corner Reflector}

\author{
N. Hata and K. Shimoda \\ Institute of Physical and Chemical Research, Wako-SHI, Saitama 351, Japan
}

Appl. Phys. 22, 1-9 (1980)

On page 6 , the second column, the 12 th line should read $=556 \mathrm{~m} / \mathrm{s}$ instead of $=278 \mathrm{~m} / \mathrm{s}$. Numerical values of the power $P$ and the signal power $\Delta P$ should therefore be multiplied by 4 , while the frequency difference $\omega-\omega_{0}$ and the gas pressure $p$ should be multiplied by 2 . Those values in Fig. 5-8 should likewise be corrected. 